\title{
Remoção de nitrogênio em sistema combinado anóxico-aeróbio com biomassa imobilizada
}

\author{
Nitrogen removal in combined anoxic-aerobic system with imobilized biomass
}

Mario Luiz Rodrigues Foco', Guilherme Pio dos Reis Lopes², Edson Aparecido Abdul Nour ${ }^{3}$

口-

\begin{abstract}
RESUMO
Este trabalho visa estudar o desempenho de um sistema combinado na remoção de matéria orgânica e nitrogênio de esgoto sanitário, por meio da recirculação interna do efluente. Os reatores, em escala de bancada, foram: filtro anóxico (32,6 L), biofiltro aerado submerso (17,6 L) e decantador secundário (7,2 L). As razões de recirculação foram 0,5; 1,5; 2,0 e 4,0 sem adição de carbono externo ou alcalinizante. Na razão de recirculação de 4,0 foram aplicadas taxas de carregamento orgânico e de nitrogênio ao

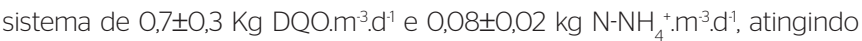
remoções de demanda química de oxigênio e nitrogênio total de $92 \pm 4 \%$ e $83 \pm 8 \%$, respectivamente. Os resultados mostram viabilidade do uso da recirculação do efluente, uma vez que as concentrações finais de

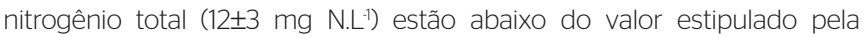
Resolução CONAMA no 430/2011.
\end{abstract}

Palavras-chave: filtro anóxico; biofiltro aerado submerso; nitrificação; desnitrificação; biomassa imobilizada; esgoto sanitário.

\begin{abstract}
This work aims to study the performance of a combined system in the removal of organic matter and nitrogen from sanitary sewage through internal recirculation of the effluent. The bench scale reactors were: anoxic filter (32.6 L), submerged aerated biofilter (17.6 L) and secondary settler (7.2 L). The recirculation rates were 0.5; 1.5; 2.0 and 4.0 without added external-carbon or alkalising. In 4.0 recirculation rate, the organic and nitrogen loading rates applied to the system were $0.7 \pm 0.3 \mathrm{~kg}$ chemical oxygen demand. $\mathrm{m}^{-3} \cdot \mathrm{d}^{-1}$ and $0.08 \pm 0.02 \mathrm{~kg} \mathrm{~N}-\mathrm{NH}_{4}{ }^{+} \cdot \mathrm{m}$ ${ }^{3} \cdot \mathrm{d}^{-1}$, reaching removals of chemical oxygen demand and total nitrogen of $92 \pm 4 \%$ and $83 \pm 8 \%$, respectively. The results show the feasibility of using recirculation of effluent, once the final concentrations of total nitrogen $\left(12 \pm 3 \mathrm{mg} \cdot \mathrm{L}^{-1}\right)$ are below the value stipulated by CONAMA Resolution no 430/2011.
\end{abstract}

Keywords: anoxic filter; aerated submerged biofilter; nitrification; nitrification; denitrification; immobilized biomass; sanitary sewage.

\section{INTRODUÇÃO}

As Estações de Tratamento de Esgoto na maior parte dos países desenvolvidos, principalmente na Europa e América do Norte, devem cumprir padrões de lançamento, que podem atingir valores abaixo de $10 \mathrm{mg} \mathrm{N} \cdot \mathrm{L}^{-1}$ para nitrogênio total (NT), como medida de proteção dos corpos receptores (REIS \& MENDONÇA, 2009). No Brasil, a Resolução CONAMA no 430/2011 determina como parâmetro regulador do lançamento de compostos nitrogenados em efluentes sanitários e industriais o nitrogênio amoniacal, sendo o valor de $20 \mathrm{mg} \mathrm{N} . \mathrm{L}^{-1} \mathrm{o}$ valor limite de referência (BRASIL, 2011).

Segundo Costa (2008), considerando-se a concentração média de nitrogênio presente no esgoto sanitário típico do Brasil, o padrão proposto pela Resolução não poderia ser atingido com a implementação dos processos de tratamento mais comumente empregados no país, tornando-se compulsória a adoção de sistemas terciários de tratamento de esgoto, sistemas mais sofisticados e onerosos. Essa imposição seria frontalmente contrária ao conceito de metas progressivas e à necessidade de universalização dos serviços de saneamento, conforme estabelecido pela Política Nacional de Saneamento (BRASIL, 2007).

Os sistemas convencionais de tratamento de esgoto, projetados visando principalmente à remoção de matéria orgânica, resultam em efluente com concentrações de nitrogênio próximas às do esgoto bruto, dificultando o atendimento às exigências do CONAMA com relação aos teores no corpo d’água (MOTA \& VON SPERLING, 2009).

Entre as opções de tratamento de esgoto sanitário, os sistemas combinados anaeróbio-aeróbio destacam-se devido a inúmeras vantagens: baixa potência de aeração requerida na fase aeróbia, menor produção de lodo biológico e baixo custo de implantação e operação,

口-

'Doutorando Engenharia Civil, Saneamento e Ambiente pela Universidade Estadual de Campinas (UNICAMP) - Campinas (SP), Brasil.

${ }^{2}$ Mestre em Engenharia Civil, Saneamento e Ambiente pela UNICAMP - Campinas (SP), Brasil.

'Doutor em Hidráulica e Saneamento pela Escola de Engenharia de São Carlos da Universidade de São Paulo (EESC/USP). Professor Associado Departamento de Saneamento e Ambiente da UNICAMP - Campinas (SP), Brasil.

Endereço para correspondência: Mario Luiz Rodrigues Foco - Avenida Oswaldo Cruz, 500 - 13083-876 - Campinas (SP), Brasil - E-mail: foco_mario@yahoo.com.br Recebido: 25/07/13 - Aceito: 25/03/14 - Reg. ABES: 1164 
quando comparados aos sistemas convencionais por lodos ativados (GONÇALVES et al., 2001; CHERNICHARO, 2006). No entanto, segundo van Haandel e van der Lubbe (2007), um inconveniente da aplicação de pré-tratamento anaeróbio é a dificuldade de posterior remoção de nitrogênio devido ao aumento da proporção nitrogênio total Kjeldhal/demanda química de oxigênio (NTK/DQO) (há remoção de material orgânico, mas não de nitrogênio) no efluente anaeróbio, causando um efeito negativo sobre a taxa de nitrificação e a capacidade de desnitrificação. Mota e von Sperling (2009) relatam que uma relação NTK/DQO igual a 0,125 é excepcionalmente elevada para o esgoto bruto e indica que a disponibilidade de material orgânico para a desnitrificação é pouca, sendo uma das razões pelas quais a desnitrificação completa se torna impossível em uma única câmara anóxica.

A utilização de filtros submersos em série para nitrificação e desnitrificação foram propostos por Jimenez et al. (1987) e Çeçen e Gonec (1992), com a seguinte configuração: parte do esgoto sanitário alimentava um primeiro filtro aeróbio e o efluente nitrificado dessa unidade era combinado com outra parte do esgoto bruto para alimentar um filtro não aerado, para remoção de matéria orgânica e nitrogênio. Os primeiros autores utilizaram esgoto sanitário com relação NTK/DQO de 0,08; vazão de alimentação $4 \mathrm{~L} \cdot \mathrm{s}^{-1}$ e razões de recirculação (R) de 2,5 alcançando eficiência de desnitrificação $\left(\mathrm{E}_{\mathrm{D} \%}\right)$ de $99 \%$, com vazão de

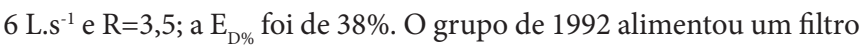
aeróbio com esgoto sintético, encaminhando o efluente nitrificado com adição de melaço na relação demanda química de oxigênio/nitrogênio oxidado (nitrito mais nitrato) $\left(\mathrm{DQO} / \mathrm{N}-\mathrm{N}_{\mathrm{OX}}\right)$ igual a 5 para um filtro anóxico, alcançando $\mathrm{E}_{\mathrm{D} \%}$ de $50 \%$, quando aplicada taxa de carregamento de nitrogênio oxidado $\left(\mathrm{N}_{\mathrm{OX}}\right)$ de $5 \mathrm{~g} \mathrm{~N}-\mathrm{N}_{\mathrm{OX}} \mathrm{m}^{-2} \cdot \mathrm{d}^{-1}$.

Simplificações foram feitas nesses sistemas para incorporar os processos anaeróbio e aeróbio em um único reator. Chui et al. (2001) operaram um único reator apresentando zonas anaeróbia, anóxica e aeróbia, alimentado com esgoto sintético com relação NTK/DQO de 0,09, Rigual 3 e temperatura de $35^{\circ} \mathrm{C}$, alcançando eficiências de remoção de DQO e NT de 98 e 90\%, respectivamente. Um reator integrado anaeróbio-aeróbio com biomassa imobilizada em escala piloto foi utilizado por Del Pozo e Diez (2005), alimentado com efluente de abatedouro de aves com NTK/DQO de 0,10 e obteve eficiências de remoção de DQO e NTK de 93 e 67\%, respectivamente, aplicando taxa de carregamento orgânico (TCO) de $0,77 \mathrm{~kg}$ DQO. $\mathrm{m}^{-3} \cdot \mathrm{d}^{-1}$ e taxa de carregamento de nitrogênio (TCN) de $0,084 \mathrm{~kg} \mathrm{~N} \cdot \mathrm{m}^{-3} \cdot \mathrm{d}^{-1}$. Nessa configuração, a desnitrificação foi de 12 a 34\%, sendo limitada pela concentração de oxigênio na zona anaeróbia, acima de $0,5 \mathrm{mg} \mathrm{O}_{2} \cdot \mathrm{L}^{-1}$, causada pela recirculação por air-lift. A maior parte do nitrogênio removido foi usado para síntese celular. Araújo Jr e Zaiat (2009) relataram a concepção de um reator combinado anaeróbio-aeróbio com filme fixo, alimentado com efluente industrial com NTK/DQO=0,11, vazão de 8 L.h ${ }^{-1}$ e R igual 3,5, resultando em TCO e TCN aplicadas de $0,97 \mathrm{~kg}$ DQO.m $\mathrm{m}^{-3} \cdot \mathrm{d}^{-1}$ e $0,11 \pm 0,01 \mathrm{~kg} \mathrm{~N} \cdot \mathrm{m}^{-3} \cdot \mathrm{d}^{-1}$, respectivamente, alcançando eficiências de remoção de DQO, NT e $\mathrm{E}_{\mathrm{D} \%}$ de 97,77 e 70\%, respectivamente.

Outra configuração é o filtro anaeróbio seguido de biofiltro aerado submerso para nitrificação e desnitrificação com recirculação de efluente, proposta por Del Pozo e Diez (2003). Utilizando efluente de abatedouro de aves com NTK/DQO igual a 0,17, variando a razão de recirculação de 1 a 6 , alcançaram eficiências de remoção de DQO de $92 \%$ com TCO aplicada de $0,39 \mathrm{~kg}$ DQO.m ${ }^{-3} \cdot \mathrm{d}^{-1}$ e de NTK de 95 e $84 \%$ com TCN aplicadas de 0,064 e $0,14 \mathrm{~kg} \mathrm{NTK} \cdot \mathrm{m}^{-3} \cdot \mathrm{d}^{-1}$, respectivamente.

Porém, os primeiros sistemas foram utilizados para tratamento de esgoto sanitário com baixas concentrações de nitrogênio (baixas razões NTK/DQO), além de elevada disponibilidade de alcalinidade, seja naturalmente presente no afluente ou adicionada. Além de estudos com esgoto sintético, com adição de fonte externa de carbono.

A proposta deste trabalho foi investigar o desempenho de um sistema combinado por reatores anóxico e aeróbio para a remoção de nitrogênio de esgoto sanitário com relação NTK/DQO desfavorável, sem adição de fonte externa de carbono ou alcalinidade, como proposta para viabilizar a nitrificação e desnitrificação em Estações de Tratamento de Esgoto combinada anaeróbia-aeróbia, atualmente em operação.

\section{METODOLOGIA}

O sistema combinado (anóxico-aeróbio), constituiu-se de um filtro anóxico (FA) de fluxo ascendente seguido por um biofiltro aerado submerso (BAS) e decantador secundário (DEC), segundo apresentado no desenho esquemático da Figura 1.

As unidades constavam de 3 reservatórios em PVC com volume útil de 32,6 L para o FA; 17,6 L para o BAS e 7,2 L para o DEC (Tabela 1).

Ambos os reatores utilizaram eletrodutos corrugados de PVC como suporte para a imobilização da biomassa, com dimensões $25,4 \mathrm{~mm}$ (diâmetro) por 15,0 mm (comprimento) e índice de vazios de 93,2 $\pm 0,3 \%$.

O fornecimento de ar ao BAS foi realizado por uma membrana difusora de microbolhas instalada no fundo falso em fluxo co-corrente com a alimentação do reator. A vazão de ar foi controlada por um rotâmetro (Dwyer, modelo RMA-21-SSV, vazão 10 L.min ${ }^{-1}$ ) e a alimentação de ar foi fornecida por um compressor (Schulz, modelo 10 pés), mantendo a concentração de oxigênio dissolvido na faixa de $5,0 \pm 0,7 \mathrm{mg} \mathrm{O} \cdot \mathrm{L}^{-1}$.

Os reatores foram dimensionados a partir de recomendações contidas no material desenvolvido pelo Programa de pesquisa em saneamento básico (PROSAB) (CAMPOS, 1999). A partir do conhecimento prévio das características do esgoto afluente, o volume do FA foi determinado fixando-se a vazão da bomba de alimentação (Tabela 2) e adotando-se um tempo de detenção hidráulico $(\mathrm{TDH})$ suficiente para alcançar em torno de $50 \%$ de remoção de DQO afluente. O volume do BAS foi 
determinado em função da TCO e da TCN, fixadas em 1,0 kg DQO. $\mathrm{m}^{-3} \cdot \mathrm{d}^{-1}$ e $0,2 \mathrm{~kg} \mathrm{~N}-\mathrm{NH}_{4}^{+} \cdot \mathrm{m}^{-3} \cdot \mathrm{d}^{-1}$, conforme a Tabela 3 .

O esgoto bruto afluente foi gerado no complexo hospitalar da Universidade Estadual de Campinas, São Paulo, que atende a uma demanda de cerca de 10 mil pessoas que diariamente circulam em suas instalações (hospital de clinicas, laboratórios, restaurantes, banco e escola). O esgoto foi coletado diariamente a partir de um poço de inspeção e limpeza da rede coletora e encaminhado para o reservatório de alimentação (tanque de equalização B) com volume de 60 L, suficiente para alimentar o sistema por $24 \mathrm{~h}$. Após esse período, o conteúdo era descartado e o reservatório lavado para receber esgoto bruto novo, mantendo sua homogeneização por meio de agitação forçada de uma bomba submersa (Sarlobetter, modelo B500).

As dimensões e características físicas de cada unidade integrante do sistema combinado (SC) estão apresentadas na Tabela 1. A alimentação com Esgoto Bruto (A1) do SC foi realizada por uma bomba peristáltica (B) (Provitec, modelo DM5000) com vazão (Q) de 2,17 L.h ${ }^{-1}$, a partir do fundo do FA. O efluente do FA (2) era encaminhado por gravidade ao fundo falso do BAS. A recirculação foi feita a partir do DEC (3) para o FA, por uma bomba de diafragma (F) (ProMinent, modelo GALA0220PVT) com vazão (q) variando conforme apresentado na Tabela 2.

Nos pontos 1, 2 e 3 (Figura 1) foram coletadas as amostras que representam, respectivamente, o esgoto bruto afluente ao sistema, o efluente anóxico (FA) e o efluente aeróbio (BAS+DEC).

O DEC foi dimensionado para uma taxa de aplicação superficial (TAS) máxima de $18,2 \mathrm{~m}^{3} \cdot \mathrm{m}^{-2} \cdot \mathrm{d}^{-1}$ (referente a uma razão de recirculação não aplicada neste trabalho de 6,0), portanto na etapa 4 a TAS foi de
13,0 $\mathrm{m}^{3} \cdot \mathrm{m}^{-2} \cdot \mathrm{d}^{-1}$. Calculou-se a TAS utilizando a vazão $(\mathrm{Q}+\mathrm{q})$, portanto variou com o aumento da vazão de recirculação (q), pois o efluente foi recirculado após o decantador.

O estudo foi dividido em quatro etapas sequenciais, cujos detalhes operacionais são apresentados na Tabela 2, com os valores médios e desvio padrão das TCO e TCN reais aplicadas ao BAS em cada etapa.

Nessas condições, o tempo de detenção hidráulica (TDH) do esgoto bruto no sistema combinado (FA+BAS+DEC) foi de 26,4 h; sendo $15,0 \mathrm{~h}$ no FA; 8,1 h no BAS e 3,3 h no DEC.

O FA foi inoculado com lodo de um filtro anaeróbio (V=300 L; $\mathrm{TDH}=12 \mathrm{~h}$; alimentado por esgoto bruto de mesma origem), após sedimentação em béquer por 15 min e remoção do sobrenadante, totali-

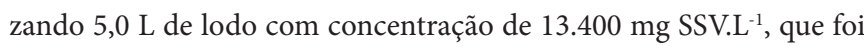
depositado no fundo falso do reator, o qual teve seu volume completado com esgoto bruto, permanecendo em repouso por $72 \mathrm{~h}$. Após esse período, as bombas foram ligadas com regime alternando entre funcionamento e repouso de 45 e 15 minutos, respectivamente.

Tabela 1 - Dimensões dos reatores do sistema combinado anóxico-aeróbio.

\begin{tabular}{|c|c|c|c|c|}
\hline Dimensões & Unidade & $\begin{array}{l}\text { Filtro } \\
\text { anóxico }\end{array}$ & $\begin{array}{l}\text { Biofiltro aerado } \\
\text { submerso }\end{array}$ & Decantador \\
\hline Altura & \multirow{2}{*}{$\mathrm{m}$} & 0,40 & 0,40 & 0,32 \\
\hline Diâmetro & & 0,39 & 0,29 & 0,19 \\
\hline Área & $\mathrm{m}^{2}$ & 0,12 & 0,07 & 0,02 \\
\hline Volume total & & 47,7 & 26,4 & 10,0 \\
\hline Volume útil & & 32,6 & 17,6 & 7,2 \\
\hline
\end{tabular}

Tabela 2 - Condições de operação do sistema combinado de tratamento em cada etapa.

\begin{tabular}{|c|c|c|c|c|c|c|c|}
\hline \multirow{2}{*}{ Etapa } & \multirow{2}{*}{ Operação (d) } & \multirow{2}{*}{ Período (d) } & Q & $q$ & $\mathrm{q} / \mathrm{Q}$ & $\mathrm{TCO}$ & $\mathrm{TCN}$ \\
\hline & & & $\left(L . h^{-1}\right)$ & $\left(L . h^{-1}\right)$ & & & \\
\hline 1 & 84 & 1 a 85 & \multirow{4}{*}{2,17} & 1,09 & 0,5 & $0,9 \pm 0,5$ & $0,20 \pm 0,02$ \\
\hline 2 & 84 & 92 a 176 & & 3,26 & 1,5 & $0,7 \pm 0,2$ & $0,22 \pm 0,06$ \\
\hline 3 & 70 & 183 a 253 & & 4,34 & 2,0 & $0,8 \pm 0,1$ & $0,22 \pm 0,07$ \\
\hline 4 & 84 & 260 a 344 & & 8,68 & 4,0 & $1,2 \pm 0,5$ & $0,18 \pm 0,09$ \\
\hline
\end{tabular}

Q: vazão afluente; q: vazão de recirculação; TCO: taxa de carregamento orgânico (kg DQO. $\left.\mathrm{m}^{-3} \cdot \mathrm{d}^{-1}\right)$; $\mathrm{TCN}$ : taxa de carregamento de nitrogênio $\left(\mathrm{kg} \mathrm{NH}_{4}{ }^{+} \cdot \mathrm{m}^{-3} \cdot \mathrm{d}^{-1}\right)$.

Tabela 3 - Condições recomendadas na literatura para favorecer a conversão de demanda química de oxigênio e ocorrência de nitrificação em biofiltro aerado submerso.

\begin{tabular}{c|c|c} 
Parâmetros & Unidade & Faixa de operação \\
\hline Carga orgânica aplicada $^{1}$ & $\mathrm{~kg} \mathrm{DQO} \cdot \mathrm{m}^{-3} \cdot \mathrm{d}^{-1}$ & $0,5-1,0$ \\
\hline Carga de amônia aplicada $^{2}$ & $\mathrm{~N}-\mathrm{NH}_{4}^{+} \cdot \mathrm{m}^{-3} \cdot \mathrm{d}^{-1}$ & $<0,20$ \\
\hline Taxa de aeração & $\mathrm{Nm}^{3} \cdot \mathrm{kg} \mathrm{DBO}_{\text {aplicada }}^{-1}$ & $>60$ \\
\hline Alcalinidade parcial & $\mathrm{mg} \mathrm{CaCO}_{3}\left({\left.\mathrm{mg} \mathrm{N}-\mathrm{NH}_{4}^{+}\right)^{-1}}^{7,07}\right.$ \\
\hline Tempo de detenção hidráulica & $\mathrm{h}$ & $3,5-8,5$ \\
\hline
\end{tabular}

'Faixa de valores para eficiência de conversão de N-amoniacal entre 50-70\%; ${ }^{2}$ recomendação TCHOBANOGLOUS et. al. (2003).

Fonte: adaptado de Mota \& von Sperling (2009). 
O sistema foi alimentado por 344 dias, no interior do laboratório, à temperatura $25 \pm 2^{\circ} \mathrm{C}$.

$\mathrm{Na}$ Tabela 3 são apresentadas as condições recomendadas na literatura em termos de taxa de carregamento de DQO, $\mathrm{N}_{-} \mathrm{NH}_{4}^{+}$e taxa de aeração e requerimento de alcalinidade para favorecimento da nitrificação em BAS.

No BAS, elevados valores de TCO irão favorecer os microrganismos heterótrofos na competição com os autótrofos por espaço (biofilme) e oxigênio, enquanto que elevados valores de TCN implicam em maior necessidade de alcalinidade para nitrificação.

Diante disso, estabeleceu-se a recirculação do efluente de forma a favorecer a nitrificação no BAS, sem adição de fonte externa de alcalinidade, e a desnitrificação no FA, sem adição de fonte externa de carbono, visando viabilizar a remoção de nitrogênio em Estações de Tratamento de Esgoto combinada anaeróbia-aeróbia, atualmente em operação, dimensionadas apenas para remoção de matéria orgânica. Foram praticadas as razões de recirculação (q/Q) de 0,$5 ; 1,5 ; 2,0$ e 4,0 .

$\mathrm{Na}$ Tabela 4 são apresentadas as variáveis, as frequências, os pontos de coleta e o método analítico empregado no monitoramento. Um balanço de massa, conforme a Equação 1, foi efetuado para corrigir o efeito de diluição refletido no efluente E2, do reator anóxico, devido à recirculação do efluente final E3, para a entrada daquele reator. As concentrações dos parâmetros monitorados reduziram significativamente, se comparados com as concentrações afluentes A1, e possibilitam interpretações equivocadas das reais eficiências e/ou processos

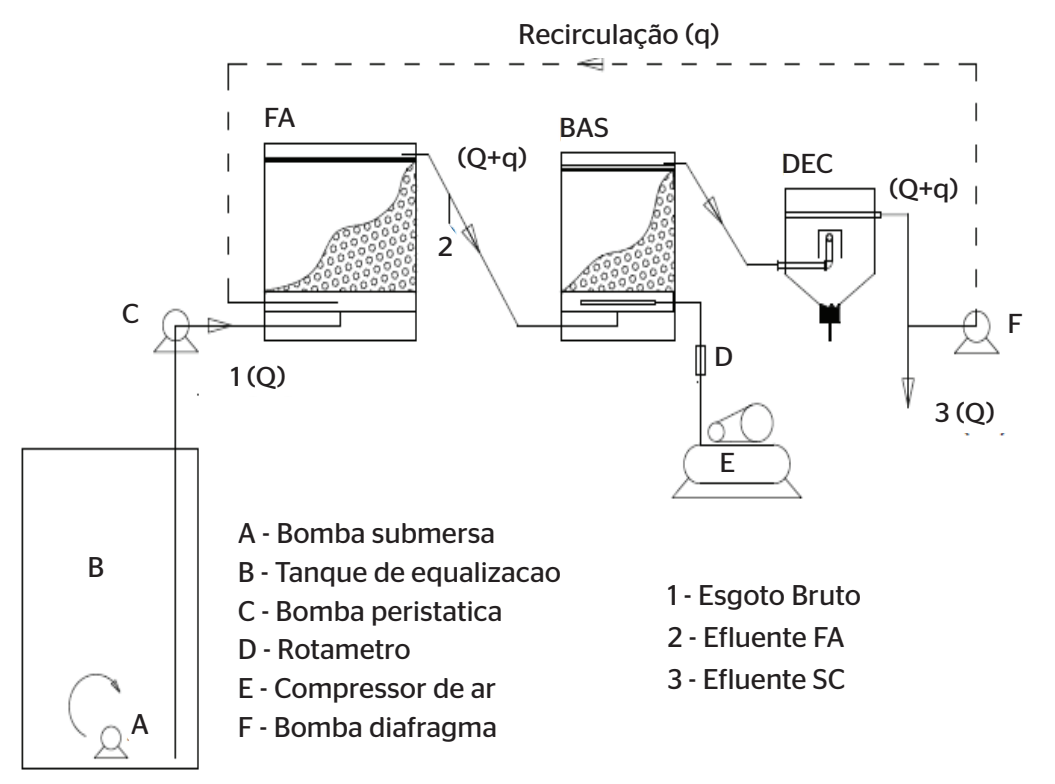

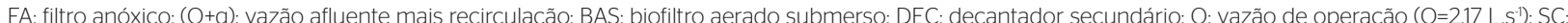
sistema combinado ( $S C=F A+B A S+D E C)$; q: vazão de recirculação, variável conforme (q/Q=0,5; 1,5; 2,0 e 4,0). Rótulo dos pontos de amostra: afluente (A1), efluente FA (E2), efluente final SC (E3).

Figura 1 - Aparato experimental do sistema combinado.

Tabela 4 - Métodos empregados nos ensaios laboratoriais e as respectivas frequências analíticas.

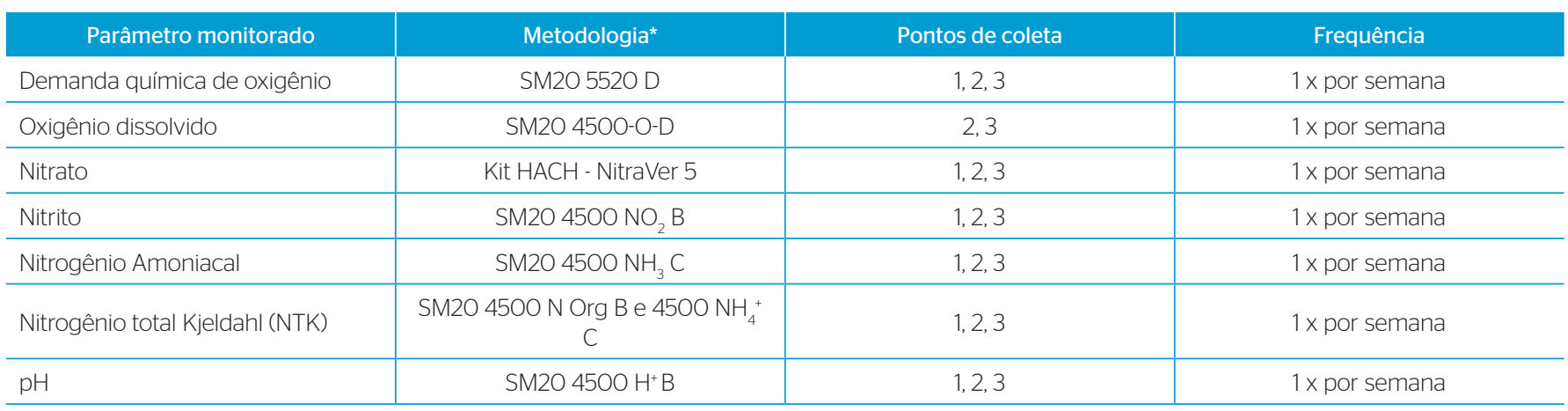

*APHA/AWWA/WEF (2012). 
que ali ocorrem. Portanto, foi inserido em cada gráfico um ponto denominado $\mathrm{A}^{\star}$, referente a esse balanço de massa.

$$
A 1^{*}=\frac{(Q \times A 1)+(q \times E 3)}{(Q+q)}
$$

Onde,

A1*: concentração diluída;

Q: vazão de projeto afluente;

A1: concentração afluente;

q: vazão de recirculação;

E3: concentração efluente final.

\section{RESULTADOS E DISCUSSÃO}

Na Tabela 5 apresenta-se a caracterização do esgoto bruto durante as quatro etapas de monitoramento. $\mathrm{O}$ sistema combinado operou por 344 dias com TCO médias de $0,7 \pm 0,3 \mathrm{~kg}$ DQO.m $\mathrm{m}^{-3} \cdot \mathrm{d}^{-1}$ e máxima de $2,0 \mathrm{~kg}$ DQO.m $\mathrm{m}^{-3} \cdot \mathrm{d}^{-1}$ (TCO aplicadas ao BAS - Tabela 2). A eficiência média de remoção de DQO foi de $91 \pm 4 \%$, conforme se pode visualizar na Figura 2.

Contudo, essas cargas são significativamente inferiores às encontradas em trabalho similar realizado por Chui et al. (2001), que reporta eficiências de remoção da ordem 98\% de DQO e 90\% de NTK para cargas aplicadas de $5 \mathrm{~kg}$ DQO. $\mathrm{m}^{-3} \cdot \mathrm{d}^{-1}$ e $0,5 \mathrm{~kg} \mathrm{~N} \cdot \mathrm{m}^{-3} \cdot \mathrm{d}^{-1}$ em sistema integrado anaeróbio-aeróbio com biomassa aderida em meio suporte randômico, com (q/Q) de 3,0. Araújo Jr e Zaiat (2009), aplicando razão de recirculação de 3,5 em um reator combinado anaeróbio-aeróbio de leito fixo, alcançaram eficiências de 97 e 77\% de remoção de DQO e NT, respectivamente, com a aplicação de TCO e TCN de $0,97 \pm 0,05 \mathrm{~kg}$ DQO.m $^{-3} \cdot \mathrm{d}^{-1} \mathrm{e}$ $0,11 \pm 0,01 \mathrm{~kg} \mathrm{NTK} \cdot \mathrm{m}^{-3} \cdot \mathrm{d}^{-1}$, respectivamente. Taxas de aplicação inferiores aos apresentados foram aplicados por Del pozo e Diez (2003) em um sistema combinando reatores anaeróbio e aeróbio em série, com (q/Q) de 6, alcançando eficiências de remoção de $92 \%$ para DQO e $84 \%$ para NTK quando as TCO e TCN foram 0,39 $\mathrm{kg}$ DQO. $\mathrm{m}^{-3} \cdot \mathrm{d}^{-1}$ e $0,14 \mathrm{~kg}$ NTK.m ${ }^{3} \cdot \mathrm{d}^{-1}$, respectivamente.

Na Figura 2 são apresentadas as concentrações de DQO afluente e efluente, as unidades e a eficiência de remoção do sistema em função do aumento da recirculação do efluente.

A carga orgânica aplicada ao FA é composta pelo afluente (A1) mais a recirculação proveniente do efluente (E3). Portanto, na Figura 2 evidencia-se a diluição na concentração de DQO, por meio da diferença entre os pontos $\mathrm{A} 1$ e $\mathrm{A} 1^{\star}$. A redução na concentração desse parâmetro foi de cerca de $30 \pm 1 ; 55 \pm 2 ; 60 \pm 4$ e $74 \pm 3 \%$ quando a razão de recirculação variou de 0,5 a 4,0, respectivamente. Em seu trabalho, Del Pozo e Diez (2003) focaram atenção na diluição do afluente ao reator anaeróbio, pois quando variaram a razão (q/Q) de 1 para 8, a concentração de DQO caiu consideravelmente (100-300 $\left.\mathrm{mg} \mathrm{O}_{2} \cdot \mathrm{L}^{-1}\right)$, tanto que a eficiência de remoção no reator se reduziu de 65 para 20\%. Os autores afirmam que isso é devido ao fato da concentração de matéria orgânica dentro do reator anóxico ter caído tanto que a cinética dos processos anóxicos e metanogênicos tornaram-se muito lentos. A eficiência do processo de desnitrificação aumenta ligeiramente com a concentração de DQO no filtro anaeróbio, enquanto que a metanogênese é mais sensível à concentração de substrato do que a desnitrificação, pois sua constante de saturação média (Ks) é de 40 a $50 \mathrm{mg}$ DQO.L ${ }^{-1}$, muito superior se comparada aos $4 \mathrm{mg}$ DQO.L $^{-1}$ para o processo anóxico. Além disso, a fração DQO recalcitrante recirculando no sistema se eleva, tornando impossível obter efluentes com DQO abaixo de 100 mg.L.-1, impactando o cálculo de eficiência.

Neste trabalho, as eficiências de remoção de DQO no FA variaram, nas quatro etapas, de $62 \pm 17 ; 62 \pm 9 ; 61 \pm 8$ e $53 \pm 15 \%$. No entanto, o aumento da recirculação do efluente no sistema não impactou a eficiência global de remoção de matéria orgânica.

Na Figura 3 mostram-se as concentrações afluente e efluente, de cada unidade, e a porcentagem de conversão de nitrogênio amoniacal no sistema.

Tabela 5 - Caracterização do esgoto bruto.

\begin{tabular}{|c|c|c|c|c|}
\hline \multirow{3}{*}{ Variável } & \multicolumn{4}{|c|}{ Razão (q/Q) } \\
\hline & 0,5 & 1,5 & 2,0 & 4,0 \\
\hline & Média $\pm \mathrm{DP}$ & Média $\pm \mathrm{DP}$ & Média $\pm \mathrm{DP}$ & Média $\pm \mathrm{DP}$ \\
\hline $\mathrm{DQO}\left(\mathrm{mg} \mathrm{O}_{2} \mathrm{~L}^{-1}\right)$ & $669 \pm 175$ & $560 \pm 92$ & $720 \pm 424$ & $719 \pm 266$ \\
\hline $\mathrm{pH}$ & $7,2 \pm 0,3$ & $7,5 \pm 0,2$ & $7,3 \pm 0,4$ & $7,0 \pm 0,6$ \\
\hline NTK (mg N.L-1) & $71 \pm 14$ & $73 \pm 9$ & $70 \pm 9$ & $81 \pm 17$ \\
\hline $\mathrm{N}-\mathrm{NH} 4+\left(\mathrm{mg} \mathrm{N} . \mathrm{L}^{-1}\right)$ & $57 \pm 11$ & $64 \pm 7$ & $59 \pm 8$ & $64 \pm 11$ \\
\hline N-org (mg N.L-1') & $14 \pm 8$ & $9 \pm 4$ & $11 \pm 4$ & $17 \pm 10$ \\
\hline Número de amostras & 13 & 13 & 13 & 11 \\
\hline
\end{tabular}

DP: desvio padrão. 


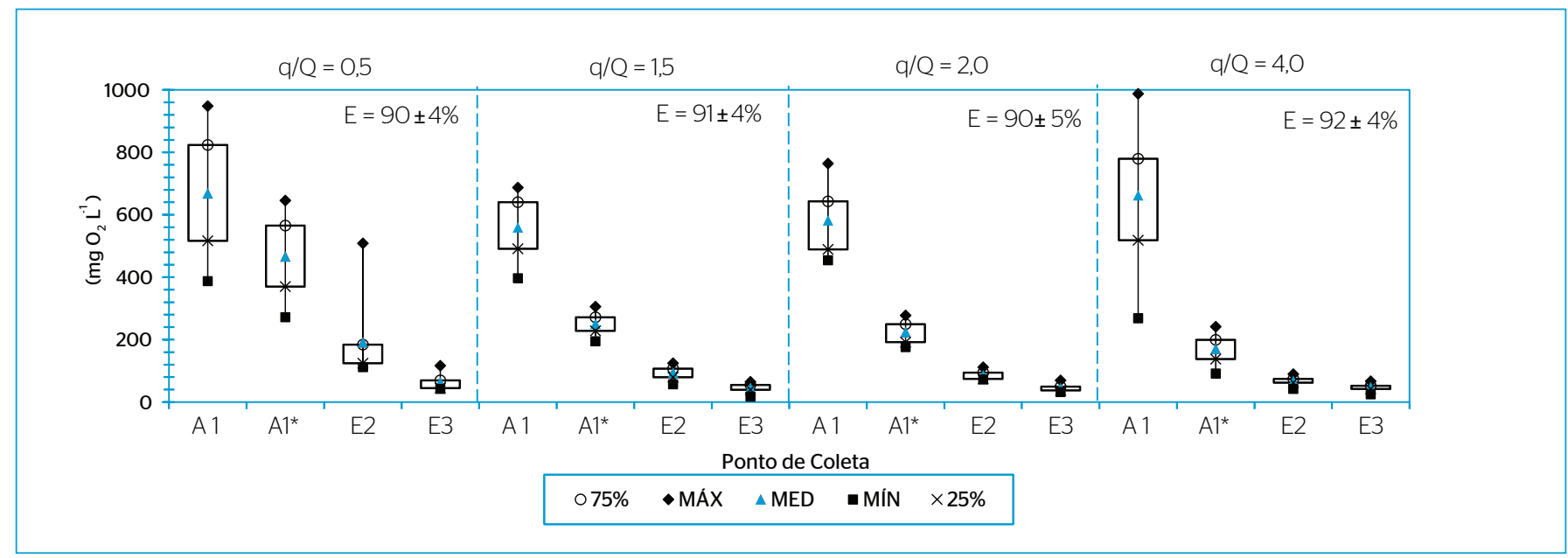

Figura 2 - Gráfico box-plot para a concentração de demanda química de oxigênio afluente (A1), diluição devido à recirculação (A1*), efluente FA (E2) e efluente final (E3). Eficiências de remoção de demanda química de oxigênio do sistema em cada etapa operacional (referente ao afluente (A1) e Efluente (E3)).

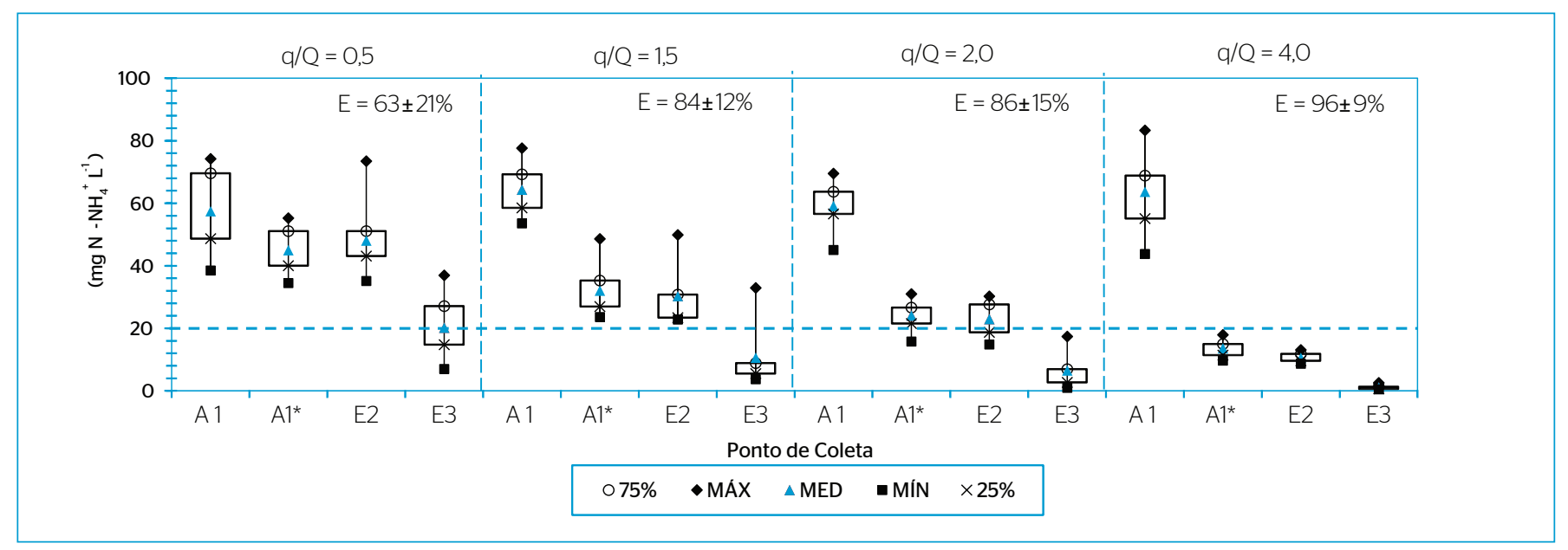

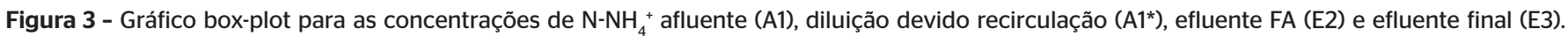
E: conversão de nitrogênio amoniacal no sistema em cada etapa operacional (referente ao afluente (A1) e Efluente (E3)). (--) meta.

Aplicou-se ao sistema combinado TCN de 0,08 $\pm 0,01 \mathrm{~kg} \mathrm{NTK} \cdot \mathrm{m}^{-3} \cdot \mathrm{d}^{-1}$, inferiores às aplicadas por Chui et al. (2001). Na Tabela 2 estão apresentadas as TCN aplicadas ao BAS durante as quatro etapas.

A eficiência de nitrificação foi superior a $50 \%$, mesmo com TCN aplicadas de $0,2 \mathrm{~kg}$ NTK.m $\mathrm{m}^{-3}$. ${ }^{-1}$, sem adição de alcalinidade complementar. O que demonstra que a recirculação do efluente proporcionou condições para aumentar a nitrificação no BAS (Figura 3).

É possível verificar, com a análise das Figuras 2 e 3, que a maior fração de DQO foi removida no FA enquanto que o nitrogênio amoniacal foi totalmente nitrificado no BAS, explicado por Del Pozo e Diez (2003) devido ao maior volume do reator anaeróbio que o aeróbio (2:1). Segundo os autores, se a concentração de 20 mg.L.'-1 de nitrogênio amoniacal ainda fosse parâmetro de qualidade para o lançamento de efluente de tratamento de esgoto sanitário (BRASIL, 2011), apenas com razão (q/Q) de 1,5 seria possível atender à legislação.

Naquela etapa, a concentração de descarte foi superada em um único dia $\left(119^{\circ}\right)$ quando um valor atípico de NTK de $84 \mathrm{mg} . \mathrm{L}^{-1}$, TCN aplicada de $0,37 \mathrm{~kg} \mathrm{~N}-\mathrm{NH}_{4}{ }^{+} \cdot \mathrm{m}^{-3} \cdot \mathrm{d}^{-1}$ no BAS, acarretou em concentração de nitrogênio amoniacal efluente de $33 \mathrm{mg} . \mathrm{L}^{-1}$, acima da meta. Excluindo esse evento, a concentração média de nitrogênio amoniacal efluente seria de $9 \pm 6 \mathrm{mg} \cdot \mathrm{L}^{-1}$.

Na Figura 4 mostra-se o gráfico box-plot apresentando as respectivas concentrações afluente e efluente às unidades do sistema combinado, e a porcentagem de remoção de NT em cada etapa.

A eficiência de remoção de nitrogênio do sistema foi calculada através da a Equação 2.

$$
N T_{\%}=\left[\frac{N T_{A 1}-N T_{E 3}}{N T_{A 1}}\right] \times 100
$$




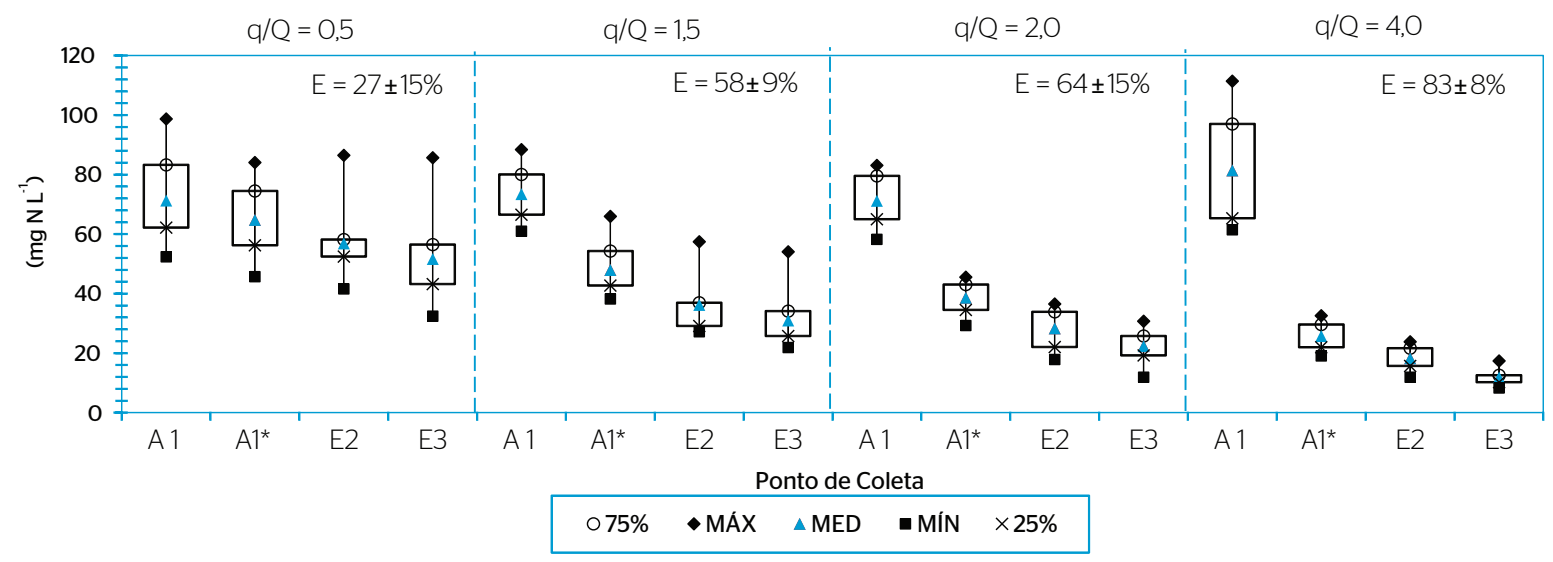

Figura 4 - Gráfico box-plot para as concentrações de nitrogênio total (NT) afluente (A1), diluição devido recirculação (A1*), efluente FA (E2) e efluente final (E3). Eficiência de remoção de NT do sistema em cada etapa operacional (referente ao afluente (A1) e Efluente (E3)).

Onde,

$\mathrm{NT}_{\%}$ : eficiência de remoção de nitrogênio total;

$\mathrm{NT}_{\mathrm{A} 1}$ : nitrogênio total afluente;

$\mathrm{NT}_{\mathrm{E} 3}$ : nitrogênio total efluente final.

Essa expressão não permite afirmar se ocorreu remoção por desnitrificação, ou se a diferença nas concentrações de entrada e saída do sistema é apenas reflexo da diluição das concentrações de NT devido à recirculação.

Em termos gerais, um balanço de nitrogênio pode ser expresso como na Equação 3.

$$
N_{\text {afluente }}-N_{\text {efluente }}=N_{\text {assimilado }}+N_{\text {desnitrificado }}
$$

O primeiro termo da Equação 3 pode ser determinado analiticamente como a concentração de NTK afluente (nitrito e nitrato afluentes ausentes). No efluente, $\mathrm{N}$ é composto por NTK mais nitrogênio oxidado $\left(\mathrm{N}_{\mathrm{OX}}\right)$ composto por nitrito + nitrato.

Com relação ao nitrogênio assimilado, é comum encontrar autores que consideram em torno de 10 a $20 \%$ da concentração afluente (CHEIBLE et al., 1993; CHUI et al., 2001). Araujo Jr e Zaiat (2009) avaliaram seu sistema sem recirculação de efluente e verificaram que $17 \%$ da concentração de NTK afluente foi removida por assimilação para síntese celular.

Os autores Jimenez et al. (1987) acrescentaram coeficientes de crescimento para bactérias aeróbias e anaeróbias em seu balanço de nitrogênio assimilado.

Assim, a estimativa do valor de NTK $_{\mathrm{OX}}$ considera que o nitrogênio afluente será removido por meio de assimilação para síntese celular em ambos reatores ou nitrificação-desnitrificação simultânea no reator aeróbio (BIESTERFELD et al., 2003). Considerando os coeficientes de rendimento celular $\mathrm{Y}_{\mathrm{AN}}$ e $\mathrm{Y}_{\mathrm{AE}}$ para bactérias anaeróbias e para aeróbias, de 0,15 e $0,5 \mathrm{mg}$ de sólidos suspensos voláteis (SSV).(mg DQO $\mathrm{REM})^{-1}$, respectivamente; e $f$ como fator de conversão de SSV para $\mathrm{N}$, igual a
0,108 mg N (mg SSV)-1 (TCHOBANOGLOUS; BURTON; STENSEL, 2003), tem-se a Equação 4.

$N T K_{o x}=\left\{\left(N T K_{A 1} \times Q\right)-\left[Y_{A N} \times\left(D Q O_{A 1}-D Q O_{E 2}\right)+Y_{A E} \times\left(D Q O_{E 2}-D Q O_{E 3}\right)\right]\right.$ $\times f \times Q\} \times 0,024$

Onde,

$\mathrm{NTK}_{\mathrm{A} 1}$ : NTK afluente;

Q: vazão afluente;

$\mathrm{Y}_{\mathrm{AN}}$ : coeficiente de rendimento celular anaeróbio;

$\mathrm{Y}_{\mathrm{AE}}$ : coeficiente de rendimento celular aeróbio;

$\mathrm{DQO}_{\mathrm{A} 1}$ : concentração de DQO afluente;

$\mathrm{DQO}_{\mathrm{E} 2}$ : concentração de DQO efluente do FA;

$\mathrm{DQO}_{\mathrm{E} 3}$ : concentração de DQO efluente final;

$f$ : fator de conversão;

0,024 fator de conversão de mg.h ${ }^{-1}$ para g.d $\mathrm{d}^{-1}$.

A partir da determinação do NTK $_{\mathrm{Ox}}$, usa-se a seguinte expressão para o cálculo da desnitrificação teórica do sistema combinado anóxico-aeróbio (Equação 5). Ou seja, o máximo valor alcançável de remoção de nitrogênio, considerando nitrificação de todo o $\mathrm{NTK}_{\mathrm{OX}}$ ' no reator aeróbio, e desnitrificação total da fração recirculada para o reator anóxico.

$$
E_{D N(\%)}=\left[\frac{N T K_{o x}-\left(N_{o x} E 3 \times Q \times 0,024\right)}{\left(N T K_{o x}\right)}\right] \times 100
$$

Onde,

$\mathrm{E}_{\mathrm{DN}(\%)}$ : máxima desnitrificação teórica;

$\mathrm{NTK}_{\mathrm{Ox}}$ : NTK oxidável;

$\mathrm{N}_{\mathrm{OX}}$ E3: concentração de nitrito + nitrato no efluente final. 
Em função dessas estimativas foi possível calcular a carga de nitrogênio assimilada, comparar com a carga total de nitrogênio removida e estabelecer a parcela de nitrogênio desnitrificado, dados apresentados na Tabela 6 .

O balanço de massa de material nitrogenado permite avaliar os procedimentos analíticos utilizados para determinar as diversas formas de nitrogênio e assegurar a quantificação das frações de nitrogênio presentes nos sistemas combinados anóxico-aeróbio.

Segundo van Haandel e Marais (1999), as frações nitrogenadas deixam o sistema sob três parcelas: assimilado no lodo, dissolvido no efluente e nitrogênio gasoso (desnitrificado). Conforme a Equação 6 (MOTA \& VON SPERLING, 2009) para o balanço de massa $\left(\mathrm{Bn}=\left(\mathrm{MN}_{\mathrm{te}}+\mathrm{MN}_{1}+\mathrm{MN}_{\mathrm{d}}\right) / \mathrm{MN}_{\mathrm{ta}}\right)$, calcula-se a massa diária de nitrogênio no efluente, a carga assimilada nos reatores para síntese celular e a carga desnitrificada (diferença da concentração na entrada e na saída do reator anóxico multiplicada pela vazão que passa por ele).

$$
\left.B n=\left\{M N_{t e}+M N_{l}+M N_{d}\right\} / M N_{t a}\right)
$$

Onde,

Bn: balanço de nitrogênio;

$\mathrm{MN}_{\mathrm{te}}$ : massa diária de nitrogênio no efluente;

$\mathrm{MN}_{\mathrm{ta}}$ : massa diária de nitrogênio no afluente;

$\mathrm{MN}_{1}$ : massa diária de nitrogênio no lodo;

$\mathrm{MN}_{\mathrm{d}}$ : massa diária de nitrogênio no desnitrificada.

Assim, conclui-se que o balanço de massa de nitrogênio fecha, com fator de recuperação $\left(B_{n}\right)$ igual a 0,97 e 0,93 para a terceira e quarta etapas, respectivamente. Os valores das duas primeiras etapas não são fidedignos, pois conforme discutido por aqueles autores, se a recuperação for menor que $90 \%$ ou maior do que $110 \%$ rejeitam-se os dados por não haver fechamento do balanço de massa. Jimenez et al. (1987) afirmam que uma diferença de até $10 \%$ entre os resultados experimentais e teóricos provavelmente é devida à hidrolise da biomassa aeróbia desprezada nos cálculos.

Uma comparação entre os resultados experimentais de remoção de NT (Equação 2) e os valores de máxima desnitrificação teórica previstos

Tabela 6 - Cargas de nitrogênio e eficiências de remoção e desnitrificação teórica em cada etapa.

\begin{tabular}{|c|c|c|c|c|c|c|c|}
\hline \multirow{3}{*}{ Etapa } & \multicolumn{7}{|c|}{ Nitrogênio } \\
\hline & Aplicado & Assimilado & Removido & Desnitrificado & Efluente & $\mathrm{NT}_{\%}$ & $\mathrm{E}_{\mathrm{DN} \%}$ \\
\hline & \multicolumn{5}{|c|}{$\left(\mathrm{g} \mathrm{N} \cdot \mathrm{d}^{-1}\right)$} & \multicolumn{2}{|c|}{$\%$} \\
\hline 1 & $3,7 \pm 0,7$ & $0,8 \pm 0,1$ & $1,1 \pm 0,6$ & $0,6 \pm 0,4$ & $2,7 \pm 0,7$ & $27 \pm 15$ & $35 \pm 8$ \\
\hline 2 & $3,8 \pm 0,5$ & $0,7 \pm 0,1$ & $2,2 \pm 0,4$ & $1,5 \pm 0,3$ & $1,6 \pm 0,5$ & $58 \pm 9$ & $62 \pm 9$ \\
\hline 3 & $3,7 \pm 0,5$ & $0,8 \pm 0,4$ & $2,5 \pm 0,4$ & $1,5 \pm 0,9$ & 1,3 $\pm 0,5$ & $64 \pm 16$ & $70 \pm 5$ \\
\hline 4 & $4,3 \pm 0,9$ & $0,9 \pm 0,4$ & $3,6 \pm 0,9$ & $2,6 \pm 0,9$ & $0,6 \pm 0,1$ & $83 \pm 8$ & $86 \pm 5$ \\
\hline
\end{tabular}

$N T_{\%}$ : eficiência de remoção de nitrogênio total; $\mathrm{E}_{\mathrm{DN} \text { \%: }}$ máxima desnitrificação teórica.

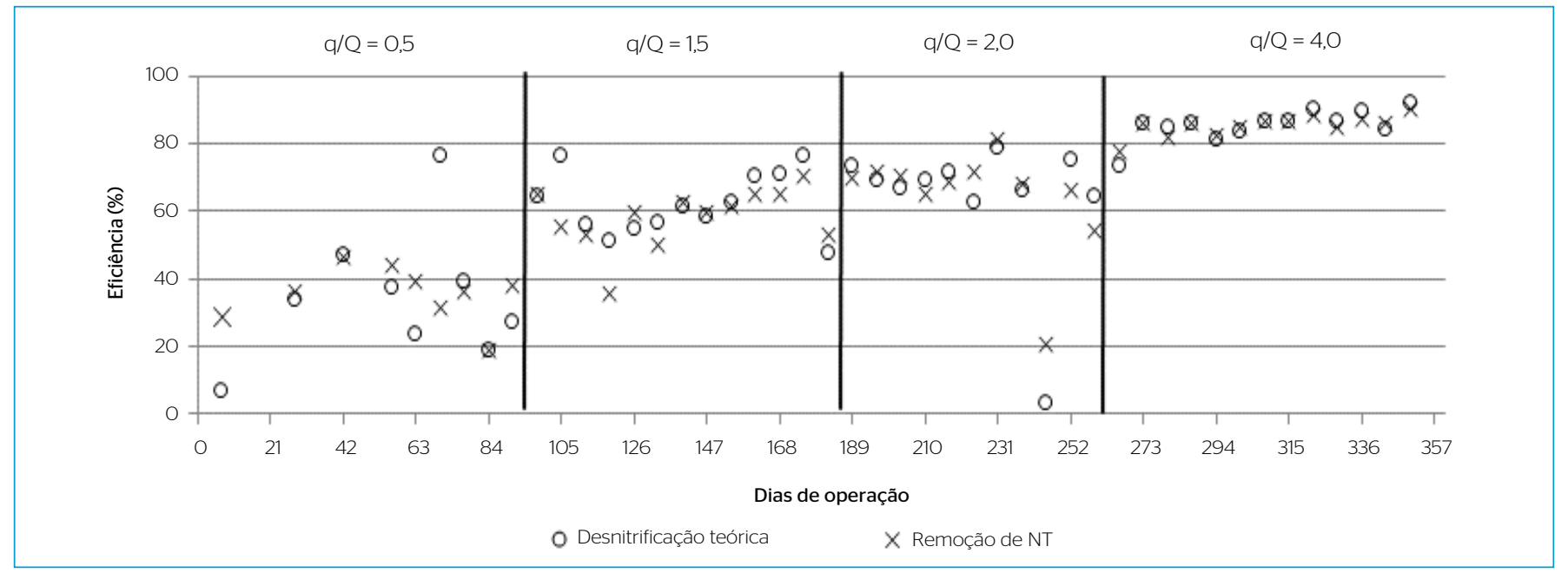

Figura 5 - Valores de desnitrificação teórica e valores experimentais de remoção de nitrogênio total obtidos para o sistema em função da razão de recirculação. 
a partir da Equação 5 são mostrados na Figura 5, com correlação de 0,93 para os resultados da quarta etapa.

Os autores Jimenez et al. (1987) e Çeçen e Gönenç (1992) afirmam que a máxima eficiência de desnitrificação só pode ser alcançada com razão DQO/ $\mathrm{N}_{\mathrm{OX}}$ de aproximadamente 5. Segundo Del Pozo e Diez (2003), a razão DQO/ $\mathrm{N}_{\text {OX }}$ no reator anaeróbio determina a competição entre os processos de metanogênese e desnitrificação, esse último é o caminho preferencial para remoção de matéria orgânica quando os valores da razão são inferiores a 10 .

Nesse trabalho, a razão $\mathrm{DQO} / \mathrm{N}_{\mathrm{OX}}$ variou em função da razão recirculação, de 16 $\pm 10 ; 5 \pm 1 ; 4 \pm 1$ e $3 \pm 1$, podendo verificar na Figura 6 que houve aumento na concentração de nitrato mais nitrito no efluente do FA, quando DQO/ $\mathrm{N}_{\mathrm{OX}}$ foi de $3 \pm 1$. Em uma avaliação com recirculação de (q/Q) 6,0 (dados não apresentados), foi inviabilizada a remoção de nitrogênio, pois os valores de $\mathrm{DQO} / \mathrm{NTK}$ e $\mathrm{DQO} / \mathrm{N}_{\text {ox }}$ tornaram-se muito baixos.

Na Figura 6 apresentam-se as concentrações das formas oxidadas de nitrogênio $\left(\mathrm{NO}_{2}^{-}\right.$e $\left.\mathrm{NO}_{3}^{-}\right)$encontradas nos efluentes E2 e E3. Em nenhuma das amostras analisadas de esgoto bruto (A1) foi detectado nitrito ou nitrato.

Quanto às concentrações de nitrito e nitrato, os dados experimentais indicam que a nitrificação e desnitrificação no sistema combinado anóxico-aeróbio com fluxo ascendente, sem recirculação de biomassa, em escala de bancada, são eficientes, visto que a concentração de nitrogênio total no efluente (Figura 4), de $11 \pm 3$ mg N.L${ }^{1}$, corresponde a apenas $15 \%$ da concentração afluente de $74 \pm 11$ $\mathrm{mg} \mathrm{N} . \mathrm{L}^{-1}$. Entretanto, a desnitrificação não foi completa no reator anóxico, evidenciada pela presença de nitrato no efluente E2
(Figura 6). Havia nitrato suficiente no reator anóxico, mostrando que sob as condições operacionais aplicadas é impossível a remoção completa de nitrato. Provavelmente devido às proporções de NTK/DQO afluente serem muito desfavoráveis $(0,125 \pm 0,04)$, que segundo Mota e von Sperling (2009), é excepcionalmente elevado para esgoto sanitário, indicando baixa disponibilidade de material orgânico para a desnitrificação.

\section{CONCLUSÕES}

A configuração se mostrou viável para alcançar a concentração de nitrogênio amoniacal de $20 \mathrm{mg} \cdot \mathrm{L}^{-1}$, com razão de recirculação de 1,5. A operação com $q / Q=4,0$ sem qualquer adição de fonte externa de carbono ou alcalinizante permitirá satisfazer as exigências de futuras legislações mais restritivas com relação à remoção de nitrogênio, uma vez que as concentrações finais de nitrogênio total

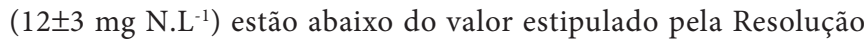
CONAMA n 430/2011.

A melhor condição operacional do sistema para desnitrificação foi obtida com razão de recirculação (q/Q) de 4,0. Nessas condições as eficiências de remoção NT e $\mathrm{E}_{\mathrm{DN} \%}$ foram de $83 \pm 8$ e $86 \pm 5 \%$, respectivamente, valores bem próximos. Porém, a desnitrificação não foi completa no reator anóxico, evidenciada pela presença de nitrato no efluente E2. Possivelmente devido à baixa relação $\mathrm{DQO} / \mathrm{N}_{\mathrm{OX}}$ de $3 \pm 1$. A relação $\mathrm{DQO} /$ NOx pode ser melhorada dosando esgoto bruto adicional diretamente no reator anóxico para complementar o requerimento de carbono da nitrificação. Entretanto, essa demanda extra e sua aplicação devem ser avaliadas para evitar deterioração da qualidade do efluente final.

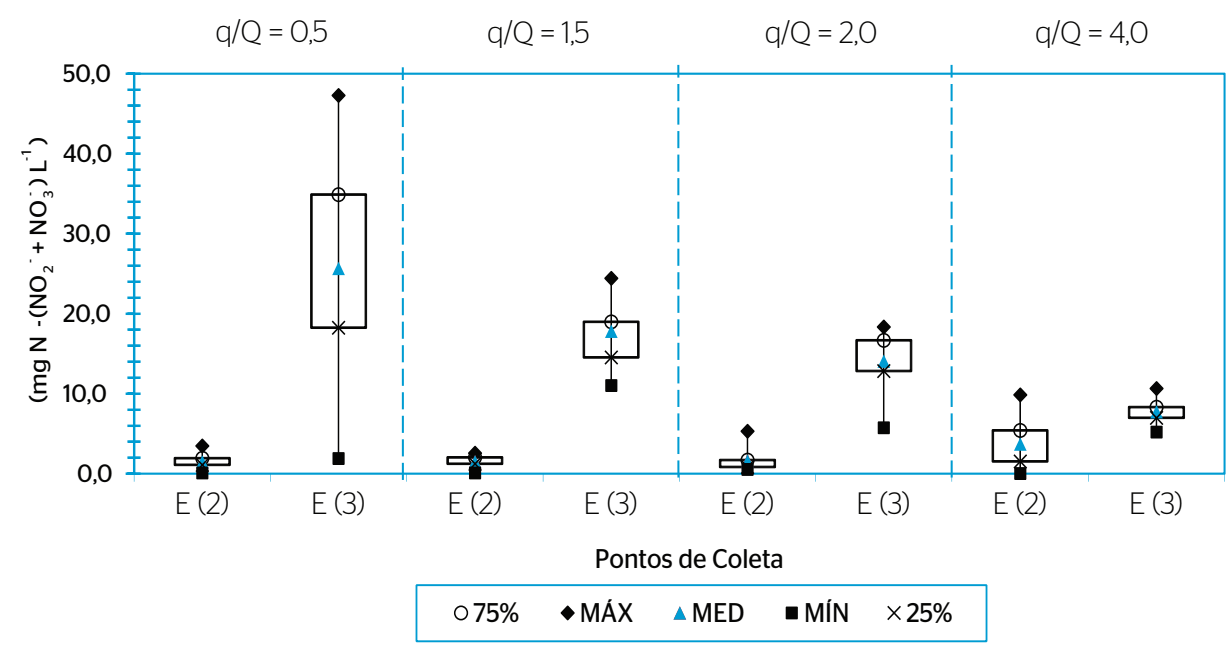

Figura 6 - Gráfico box-plot para as concentrações de nitrito e nitrato nos efluentes do FA (E2) e efluente final (E3) do sistema combinado anóxicoaeróbio. Ponto (A1) ausente, pois continha somente nitrogênio nas formas orgânica e amoniacal. 


\section{REFERÊNCIAS}

APHA/AWWA/WEF - American Public Health Association/American Water Works Association/Water Environment Federation (2012) Standard Methods for the Examination of Water and Wastewater. 22 ed. New York: APHA.

ARAUJO JR, M.M. \& ZAIAT, M. (2009) An upflow fixed-bed anaerobicaerobic reactor for removal of organic matter and nitrogen from L-Lysine plant wastewater. Canadian Journal of Civil Engineering, v. 36, p. 1085-1094.

BIESTERFELD, S.; FARMER, G.; RUSSELL, P.; FIGUEROA, L. (2003) Effect of alkalinity type and concentration on nitrifying biofilm activity. Water Environment Research, v. 75, n. 3, p. 196-204.

BRASIL. (2007) Lei no 11.445, de 5 de janeiro de 2007. Estabelece diretrizes nacionais para o saneamento básico; altera as leis no 6.766, de 19 de dezembro de 1979, no 8.036, de 11 de maio de 1990, no 8.666, de 21 de junho de 1993, no 8.987, de 13 de fevereiro de 1995; revoga a lei no 6.528, de 11 de maio de 1978 e dá outras providências. Diário Oficial da União, 8 de janeiro de 2007. p. 3-7.

BRASIL. (2011) Resolução CONAMA no 430/2011, de 13 de maio de 2011. Dispõe sobre as condições e padrões de lançamento de efluentes, complementa e altera a Resolução n 357, de 17 de março de 2005 , do Conselho Nacional do Meio Ambiente - CONAMA. Diário Oficial da União, n 92, 16 de maio de 2011. p. 89

CAMPOS, J.R. (1999) Tratamento de esgoto sanitário por processo anaeróbio e disposição controlada no solo. Rio de Janeiro: ABES - PROSAB. p. 464.

CHERNICHARO, C.A.L. (2006) Post-treatment options for the anaerobic treatment of domestic wastewater. Reviews in Environmental Science and Bio/Technology, v. 5, p. 73-92.

CHUI, P.C.; TERASHIMA, Y.; TAY, J.H.; OZAKI, H. (2001) Wastewater treatment and nitrogen removal using submerged filter systems. Water Science and Technology, v. 43, n. 1, p. 225-232.

COSTA, B.S. (2008) Decisão do CONAMA foi pelo bom senso. Abes Informa, n. 67, p. 4.

ÇEÇEN, F. \& GÖNENÇ, E. (1992) Nitrification-denitrification of highstrength nitrogen wastes in two up-flow submerged filters. Water Science and Technology, v. 26, n. 9-11, p. 2225-2228.
DEL POZO, R. \& DIEZ, V. (2003) Organic matter removal in combined anaerobic-aerobic fixed-film bioreactors. Water Research, v. 37, n. 15, p. 3561-3568.

DEL POZO, R \& DIEZ, V. (2005) Integrated anaerobic-aerobic fixed-film reactor for slaughterhouse wastewater treatment. Water Research, v. 39, n. 6, p. 1114-1122.

GONÇALVES, R.F.; CHERNICHARO, C.A.L.; ANDRADE NETO, C.O.; SOBRINHO, P.A.; KATO, M.T.; COSTA, R.H.R.; AISSE, M.M.; ZAIAI, M. (2OO1) Pós-tratamento de efluentes de reatores anaeróbios por reatores de biofilme. In: CHERNICHARO, C.A.L. (Coord.) Pós-tratamento de efluentes de reatores anaeróbios. Belo Horizonte: Projeto PROSAB 2. p. 171-278.

JIMENEZ, B.; CAPDEVILLE, B.; ROQUES, H.; FAUP, G.M. (1987) Design considerations for a nitrification-denitrification process using two fixed-bed reactors in series. Water Science and Technology, v. 19, n. 1-2, p. 139-150.

MOTA, S. \& VON SPERLING, M. (2009) Nutrientes de esgoto sanitário: utilização e remoção. vol. 1. Rio de Janeiro: PROSAB/FINEP. 430 p.

REIS, J.A.T. \& MENDONÇA, A.S.F. (2009) Análise técnica dos novos padrões brasileiros para amônia em efluentes e corpos d'água. Engenharia Sanitária e Ambiental, v. 14, n. 3, p. 353-362.

SCHEIBLE, O.K.; MULBARGER, M.; SUTTON, P.; SIMPKIN, T.; DAIGGER, G. (1993) Nitrogen Control Manual EPA/625/R-93/010. Lancaster: Technomic Pub. Co. 311 p.

TCHOBANOGLOUS, G.; BURTON, F.L.; STENSEL, H.D. (2003) Wastewater Engineering: treatment and reuse. New York: McGrawHill. $1819 \mathrm{p}$

VAN HAANDEL, A.C. \& MARAIS, G.V.R. (1999) O comportamento do Sistema de lodo ativado: teoria e aplicações para operação e projetos. Campina Grande: Epgraf. 472 p.

VAN HAADEL, A. \& VAN DER LUBE, J. (2007) Handbook biological wastewater treatment design and optimization of activated sludge systems. Leidschendam: Quist Publishing. 570 p. 